The new Breathe feature where we give you an expert and a topic, and you have the chance to ask them any questions you wish via breathe@ersj.org.uk

See page 327 for next month's expert and subjects.

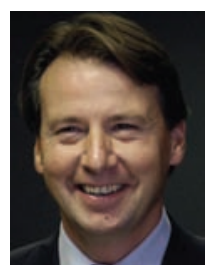

R. Gosselink

Respiratory Rehabilitation Division Universitaire Ziekenhuizen Leuven Leuven Belgium
CrossMark $\leftarrow$ click for updates

\section{Ask the expert: Exercise and rehabilitation}

Q1. We are conducting a research project with chronic obstructive pulmonary disease (COPD) patients, whom we are submitting to a cycle ergometer endurance training programme. We would like to know your opinion about the maximum work rate in the incremental test.

D. Soares (Minas Gerais, Brazil)

Intensity of training is an important parameter in obtaining training effects [1, 2]. In most studies on training in COPD patients, the target training intensity has been based on a percentage of the obtained maximal workload during an incremental exercise test [2]. However, some authors reported that patients were unable to obtain the indicated intensity of $70 \%$ of the maximal workload [3], while others trained patients with a similar degree of airflow obstruction without mentioning such complaints [4]. The maximal workload in the last step of the incremental exercise test is often not described in detail in the literature, but most investigators and clinicians will consider a minimum duration of $20 \mathrm{~s}$. However, a more important confounding factor in using maximal workload as indicator of maximal exercise capacity is its dependence on the size of the increments during an incremental exercise test [5]. The size of the steps affects the obtained maximal workload, but not the maximal oxygen uptake $\left(\mathrm{V}_{2}\right.$, $\left.\mathrm{max}\right)$ [5]. This explains the discrepant observations in the literature on the tolerance of high-intensity training.

The recommendation for the intensity of exercise training in COPD patients [2] is to standardise the protocol of the maximal exercise testing (i.e. increments in work rate) in your lab, start the exercise training protocol with a workload of $60 \%$ of maximum (obtained during the last step of the maximal exercise test for $\geq 20 \mathrm{~s}$ ) and, importantly, adapt the training intensity based upon the modified Borg scale for dyspnoea (4-6 on a 1-10 scale) [6].

Q2. If testing maximal exercise capacity in asthmatics without the benefit of a full laboratory, would the Balke protocol on a treadmill and the time achieved (T) be a useful outcome measure in a research situation? If not, what would be suitable? Are the following equations to derive an estimated $\mathrm{VO}_{2}$, max from this time useful?

Men: $V_{2} \mathrm{O}_{2}$ max $=1.44 \mathrm{~T}+14.99 ;$ Women: $V \mathrm{O}_{2}, \max =1.38 \mathrm{~T}+5.22$

I am looking at exercise tolerance outcomes in asthmatics post-breathing control techniques intervention.

\section{G. Austin (Stevenage, UK)}

Maximal exercise tests for assessing the outcome of interventions are less sensitive than the endurance type of exercise testing or field tests. The estimation of $\mathrm{V}^{\prime} \mathrm{O}_{2}$, max from simple regressions in patient populations is probably incorrect and inaccurate, due to other limitations of exercise performance than in the healthy population. For the evaluation of an intervention I would recommend measuring exercise tolerance using a constant work rate test on a bike [7], or a field test such as the shuttle walk test [8] or the 6-minute walk test (6-MWT). Details of the 6-MWT were recently summarised in a consensus document [9]. Normal values are available for the 6-MWT [10], but not for the shuttle walk test. The potential disadvantage of the 6-MWT in this population is a ceiling effect of the outcome that will be around $700 \mathrm{~m}$. In patients with better preserved exercise performance, the test might be less sensitive to treatment effects. 
Q3. Is there evidence that different exercise modalities should be applied to patients based upon lung function (forced expiratory volume in one second (FEV1)) or other measures of severity? Is there evidence of patients of different phenotypes in COPD (e.g. underweight emphysematous patients or overweight bronchitic patients) responding differently to exercise training?

A. Burnett (Rotherham, UK)

Baseline measures, especially (maximal) exercise testing with electrocardiogram, oxygen saturation and ventilatory measures, provide important information on the safety of exercise training, appropriate targeting of training intensity and factors limiting exercise performance. Information from peripheral and respiratory muscle testing might be helpful in adjusting the exercise training programme. A flowchart of reasons for exercise limitation and suggestions for content of the rehabilitation programme can be found in [11]. Although the chart has "face validity" to compose a training programme, the inclusion of the measures has limited value in selecting COPD patients who will respond to rehabilitation, including exercise training. Patients with peripheral muscle weakness and less severe ventilatory limitation are better responders. However, these factors explained only $32 \%$ of the variance in training response [12]. FEV1 is not of value in selecting patients, but in our clinical experience, most patients with FEV1 $<40 \%$ predicted will be unable to perform endurance exercise training; they will, however, be successful in performing interval training. The patients are able to obtain similar training effects with fewer symptoms of dyspnoea during the training [13]. There is some data to suggest that the addition of inspiratory muscle training (IMT) will be effective in patients with poor exercise performance and respiratory muscle weakness (maximal inspiratory pressure $(P 1$, max $\left.)<60 \mathrm{cmH}_{2} \mathrm{O}\right)$ [14]. COPD patients with peripheral muscle weakness improved to a similar extent with resistance muscle training compared with whole-body exercise [15]. The addition of resistance training to wholebody exercise had an additional effect when compared with both modalities separately [16].

Q4. What standards are generally used for evaluating the results of the 6-MWT and what size patient sample is needed to prove significant results in a pulmonology rehabilitation programme? L. Litskevich (Belarus)

Standards for the 6-MWT are provided in a recent American Thoracic Society statement [9]. We recommend encouraging patients during the test and our normal values are based on this standardisation [10]. At present, sufficient data are available to provide pulmonary rehabilitation to COPD patients and withholding this treatment for controlled trials can be considered unethical [2]. "Benchmarks" such as the minimally clinically important difference for the 6-MWT (54 m) and the health-related quality of life (Chronic Respiratory Disease Questionnaire: 10 points) can be helpful in proving that your programme is effective.

Q5. How do you select COPD patients who are likely to benefit from proportional assist ventilation in lower limb exercise training? How effective is such an approach in your experience?

Regarding the application of IMT in COPD rehabilitation, how do you select patients, and what are the outcomes in your patients who have had IMT?

K.C. Wong (Hong Kong, China)

Ventilatory limitation is a common reason for exercise limitation in patients with advanced lung disease. The options are either to reduce the load on the inspiratory muscles during exercise training by noninvasive ventilation (NIV), or to increase the capacity of the inspiratory muscles by muscle training. Both options have been studied, revealing that patients with inspiratory muscle weakness ( $P$, max $<60 \mathrm{cmH}_{2} \mathrm{O}$ ) are more likely to benefit from either treatment in terms of acute [17] or long-term [14, 18] improvement in exercise tolerance. There is no study available that compares the relative efficacy of the two treatment modalities. Since NIV is demanding for the patient, needs close supervision and expensive equipment, the option of choice in clinical practice might be IMT [19].

Q6. Do you have any guidance on the commencement of some level of rehab following acute exacerbation of COPD as an in-patient or, once home, with an early discharge scheme, to use this early period as "prehab" prior to starting a full programme? Do you have any advice on the optimum combination of endurance with strengthening exercises within an individual session?

W. Sullivan (Blackburn, UK) 
Acute exacerbation of COPD with hospitalisation results in short- and long-term physical inactivity [20] and muscle weakening [21]. This inactivity is prognostic for rehospitalisation [22], so has to be addressed early on during hospital admission. General exercise training results in large increases in metabolic requirements and consequently a high ventilatory requirement. The further reduced ventilatory capacity, due to the exacerbation, will result in early ventilatory limitation and significant dyspnoea during exercise. Endurance exercise training is thus often badly tolerated. Exercising small muscle groups (resistance training) is more appropriate with lower metabolic and ventilatory requirements. We performed a randomised controlled trial with early resistance training of lower limbs during hospitalisation. This resulted in improved muscle strength compared with the control group [23]. Furthermore, it has been shown that post-acute exacerbation rehabilitation is effective [24]. In clinical practice, we start with resistance muscle training and progress to interval training towards discharge. The programme is continued after discharge, in exercise duration as well as intensity and training modalities. Stair climbing and upper limb exercise are often not tolerated in the early phase of the postexacerbation rehabilitation, but are added in the outpatient programme later on.

We combine muscle resistance training with general exercise training (walking, cycling). Data support this combination, enhancing the outcome on performance and muscle strength [16]. Details on the optimal combination of repetitions and intensity are not available. In our programme, resistance training is applied with loads of $70 \%$ onerepetition maximum, eight repetitions and three series, and whole-body exercise is performed at 60\% of maximal workload for 25-30 min (walking and cycling).

\section{References}

1. Casaburi R, Patessio A, Ioli F, Zanaboni S, Donner CF, Wasserman K. Reductions in exercise lactic acidosis and ventilation as a result of exercise training in patients with obstructive lung disease. Am Rev Respir Dis 1991; 143: 9-18.

2. Troosters $T$, Casaburi $R$, Gosselink R, Decramer M. Pulmonary rehabilitation in chronic obstructive pulmonary disease. Am J Respir Crit Care Med 2005; 172: 19-38.

3. Maltais F., Leblanc $P$, Jobin J, et al. Intensity of training and physiologic adaptation in patients with chronic obstructive pulmonary disease. Am J Respir Crit Care Med 1997; 155: 555-561.

4. Casaburi R., Porszasz J, Burns MR, Carithers ER, Chang RS, Cooper CB. Physiologic benefits of exercise training in rehabilitation of patients with severe chronic obstructive pulmonary disease. Am J Respir Crit Care Med 1997; 155: $1541-1551$.

5. Debigare R., Maltais F, Mallet M, Casaburi R, Leblanc $P$. Influence of work rate incremental rate on the exercise responses in patients with COPD. Med Sci Sports Exerc 2000; 32: 1365-1368.

6. Horowitz MB, Littenberg B, Mahler DA. Dyspnea ratings for prescribing exercise intensity in patients with COPD. Chest 1996; 109: 1169-1175.

7. Van 't Hul A, Gosselink R, Kwakkel G. Constant-load cycle endurance performance: test-retest reliability and validity in patients with COPD. J Cardiopulm Rehabil 2003; 23: 143-150.

8. Singh SJ, Morgan MDL, Scott S, Walters D, Hardman AE. The development of the shuttle walking test of disability in patients with chronic airways obstruction. Thorax 1992; 47: 1019-1024.

9. American Thoracic Society. ATS Statement: Guidelines for the six-minute walking test. Am J Respir Crit Care Med 2002; 166: 111-117.

10. Troosters T, Gosselink R, Decramer M. Six minute walking distance in healthy elderly subjects. Eur Respir J 1999; 14: $270-274$.

11. Bekkering GE, Hendriks HJM, PatersonWJ, et al. Guidelines for physiotherapeutic management in chronic obstructive pulmonary disease (COPD). Physical Therapy Reviews 2000; 5: 59-74.

12. Troosters $T$, Gosselink $R$, Decramer M. Exercise training: how to distinguish reponders from non-responders. $J$ Cardiopulm Rehabil 2001; 21: 10-17.

13. Vogiatzis I, Nanas s, Roussos C. Interval training as an alternative modality to continuous exercise in patients with COPD. Eur Respir J 2002; 20: 12-19.

14. Lotters F, van Tol B, Kwakkel G, Gosselink R. Effects of controlled inspiratory muscle training in patients with COPD: a metaanalysis. Eur Respir J 2002; 20: 570-576.

15. Spruit MA, Gosselink R, Troosters T, De Paepe K, Decramer M. Resistance versus endurance training in patients with COPD and peripheral muscle weakness. Eur Respir J 2002; 19: 1072-1078.

16. Bernard S, Whittom F, Leblanc $P$, et al. Aerobic and strength training in patients with chronic obstructive pulmonary disease. Am J Respir Crit Care Med 1999; 159: 896-901.

17. Probst VS, Troosters T, Pitta F, Decramer M, Gosselink R. Cardiopulmonary stress during exercise training in patients with COPD. Eur Respir J 2006; 27: 1110-1118.

18. Van 't Hul A, Gosselink R, Hollander P, Postmus P, Kwakkel G. Acute effects of inspiratory pressure support during exercise in patients with COPD. Eur Respir J 2004; 23: 34-40.

19. Van 't Hul A, Gosselink R, Hollander P, Postmus P, Kwakkel G. Training with inspiratory pressure support in patients with severe COPD. Eur Respir J 2006; 27: 65-72.

20. McConnell AK, Romer LM, Weiner P. Inspiratory muscle training in obstructive lung disease. Breathe 2005; 2: 38-49.

21. Pitta F, Troosters T, Probst VS, Spruit MA, Decramer M, Gosselink R. Physical activity and hospitalization for exacerbation of COPD. Chest 2006; 129: 536-544.

22. Spruit MA, Gosselink R, Troosters $T$, et al. Muscle force during an acute exacerbation in hospitalised patients with COPD and its relationship with CXCL8 and IGF-I. Thorax 2003; 58: 752-756.

23. Garcia-Aymerich J., Lange P, Benet M, Schnohr P, Anto JM. Regular physical activity reduces hospital admission and mortality in chronic obstructive pulmonary disease: a population-based cohort study. Thorax 2006; 62: 772-778.

24. Probst VS, Troosters T, Celis G, Pitta F, Decramer M, Gosselink R. Effects of resistance exercise training during hospitalisation due to acute exacerbation of COPD preliminary results. Eur Respir J 2005; 49: 432s.

25. Man WD, Polkey MI, Donaldson N, Gray BJ, Moxham J. Community pulmonary rehabilitation after hospitalisation for acute exacerbations of chronic obstructive pulmonary disease: randomised controlled study. BMJ 2004; 329: 1209. 